\title{
Coordination of degrees of freedom and stabilization of task variables in a complex motor skill: expertise-related differences in cello bowing
}

\author{
Julius Verrel · Steven Pologe $\cdot$ Wayne Manselle • \\ Ulman Lindenberger $\cdot$ Marjorie Woollacott
}

Received: 30 July 2012/Accepted: 15 October 2012/Published online: 30 October 2012

(C) Springer-Verlag Berlin Heidelberg 2012

\begin{abstract}
Stringed instrument bowing is a complex sensorimotor skill, involving fine regulation of bow orientation and motion relative to the string. In this study, we characterize this skill in terms of stabilization of specific bow parameters as well as the underlying use and coordination of the degrees of freedom (DOF) of the right bowing arm. Age-matched samples of 10 advanced cellists and 10 cello novices took part in the study. Kinematic bow movement data were analyzed with respect to task variables suggested by the cello teaching literature: position and orientation of the bow relative to the string, bow velocity, and timing. Joint motion of the bowing arm was analyzed in terms of movement amplitude and inter-joint coordination (principal component analysis). As expected, novices showed poorer control of bowing parameters. In addition, novices differed markedly from advanced players in the use and coordination of the DOF of the bowing arm, with the elbow and wrist showing less overall movement and a reduced proportion of variance explained by the first principal component (PC1). In contrast, larger amounts of shoulder variance were explained by $\mathrm{PC} 1$ in novices compared to experts. Our findings support Bernstein's theory of graded skill acquisition, according to which early stages of motor
\end{abstract}

\footnotetext{
J. Verrel $(\bowtie) \cdot$ U. Lindenberger

Center for Lifespan Psychology, Max Planck Institute for

Human Development, Berlin, Germany

e-mail: verrel@mpib-berlin.mpg.de

S. Pologe

School of Music, University of Oregon, Eugene, OR, USA

W. Manselle $\cdot$ M. Woollacott

Department of Human Physiology and Institute of

Neurosciences, University of Oregon, Eugene, OR, USA
}

skill learning are characterized by a "freezing" of movement DOF, while later learning stages exploit the DOF, possibly following a proximal-to-distal sequence, for improved task performance.

Keywords Motor skill - Expertise - Coordination · Degrees of freedom $\cdot$ Music performance $\cdot$ Cello

\section{Introduction}

According to Bernstein's (Bernstein 1967) theory of graded skill acquisition, early stages of skill acquisition are associated with "freezing" some biomechanical degrees of freedom (DOF, e.g., joint angles). Conversely, later (higher) stages are characterized by a more differentiated use of DOF ("freeing"), allowing more efficient and flexible/functional performance. This view is supported by the studies of a variety of motor tasks, such as pistol shooting (Arutyunyan et al. 1969), hand writing (Newell and Van Emmerik 1989), racketball (Southard and Higgins 1987), or a whole-body ski-simulator task (Vereijken et al. 1992). Some of these studies (Newell and Van Emmerik 1989; Southard and Higgins 1987) found evidence for a proximal-distal gradient, with differentiated use of the distal degrees of freedom appearing later in practice.

As a complex coordinative skill, acquired through years of dedicated practice, string instrument bow technique is an excellent model for addressing general questions about motor coordination and its acquisition (Bernstein 1967, 1996). In bowed string instruments, sound is usually produced by moving the bow continuously across the string with the right arm (Fig. 1). The friction between bow and string induces vibration of the string, which is amplified by the corpus of the instrument. Cello teachers instruct students 


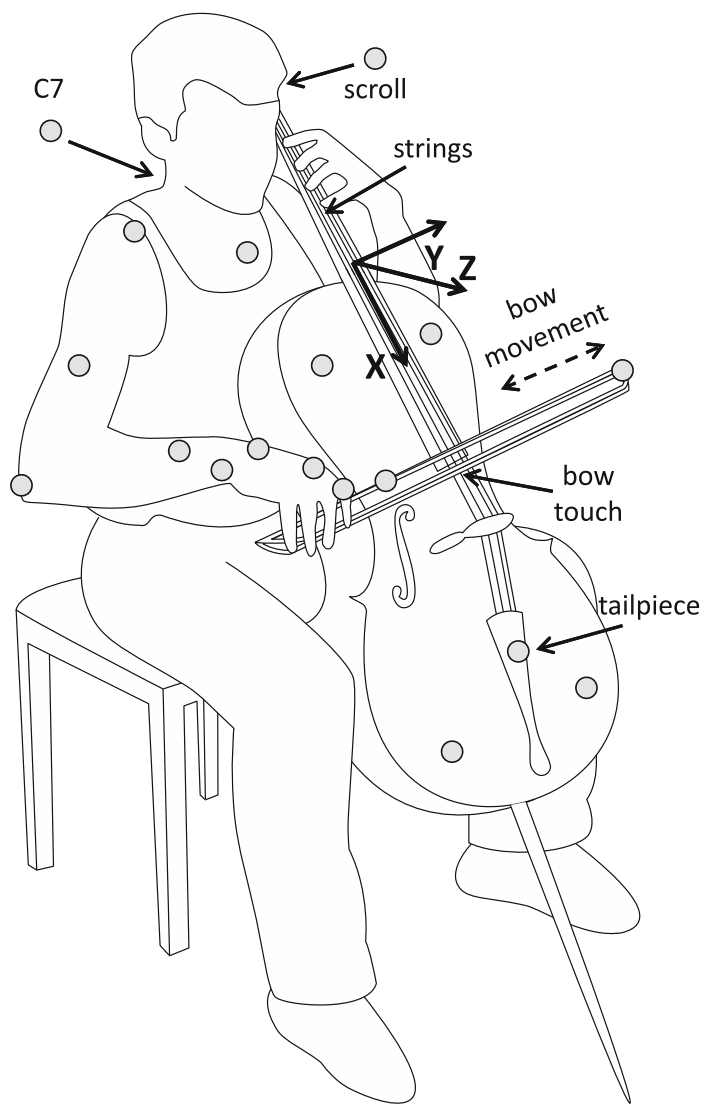

Fig. 1 Cello bowing. Illustration of setup, instrument, and task. Marker positions are indicated as gray circles. The cello-centered coordinate system was defined by the longitudinal axis from scroll to tailpiece markers $(X)$, and the direction of the lower two markers on the cello body by a Gram-Schmidt procedure. The approximate directions of the axes are indicated (note that the actual origin was defined to be at the scroll marker). Bow Direction was defined by the line through the two bow markers. The bow/string touch position was defined as the $X$ coordinated of the intersection point between the cello's XZ plane and the bow longitudinal axis. The bowing angle was defined as the angle between the longitudinal axes of bow and the cello

to keep the angle between bow and string approximately orthogonal and bowing velocity constant during the bowing movement, presumably to ensure consistently high tone quality (Mantel 1995, p. 172). Stabilizing these parameters is likely to depend on the differentiated and coordinated use of the degrees of freedom (joint angles) of the right arm. Indeed, according to the cello technique literature (Mantel 1995), proximal joints (shoulder and elbow) are crucial for the main transport movement while distal joints may play an important role for fine-tuning the movement, such as maintaining a stable bowing angle. Moreover, cello teachers emphasize the importance of achieving "freedom" in the wrist and hand during bowing (Mantel 1995). "Most beginners play with the entire forearm with no sign of wrist flexibility" (Starr 1996).
Thus, both theoretical considerations and empirical evidence from practitioners/teachers suggest that acquisition of stringed instrument bowing is associated with increased use and coordination in particular of distal DOF of the bowing arm. However, to our knowledge, there is limited experimental evidence to support this view. In fact, a recent study on violin bowing (Konczak et al. 2009) concluded that higher skill may be associated with "freezing" of DOF in violin bowing. In the Discussion section, we will provide a more detailed consideration why those authors may have reached that conclusion. Importantly, the term "freezing," while intuitively reflecting subjective experience and external observation, still lacks precise operationalization. Some authors have used the mere amount of movement at individual joints as evidence for freeing or freezing (Konczak et al. 2009). In contrast, other studies have considered both movement amplitudes and coordination patterns (e.g., cross-correlations and principal component analysis (PCA)) for more complete characterization of movement coordination (e.g., Newell and Van Emmerik 1989; Vereijken et al. 1992).

The present study aims at characterizing skilled bowing movements in cello playing and the underlying joint coordination. Skilled performance in advanced and professional cellists ("experts") was compared to performance of novices, who had only received a short basic introduction (20 min) to cello bowing and had no prior experience with bowed string instruments. We expected experts to show better control of task-relevant parameters, such as bowing angle, position of bow on the string, and bow movement velocity and duration. With respect to the use of DOF of the right (bowing) arm, we predicted increased use (movement amplitude) and coordination (component loadings and variance explained, based on PCA in experts versus novices, in particular for the more distal joints.

\section{Methods}

\section{Participants}

Ten advanced or professional players ( 3 female, age \pm SD: $22.9 \pm 4.3$ years, age range: $19-32$ years) and ten novice players ( 3 female, $23.5 \pm 3.5$ years, age range $21-32$ years) took part in the study. Advanced or professional players ("experts") had at least 5 years of cello education (12.4 \pm 5.5 years, range: $5-20$ years), at least 10 years of total cello experience (14.4 \pm 5.1 years, range: $10-24$ years), and were students of cello at a conservatory or advanced amateurs. Novices had no prior experience with the cello or any other bowed string instrument. The experiment was approved by local ethics committees (Max Planck Institute for Human Development, Berlin, and 
University of Oregon, Eugene) and conducted with participants' written informed consent and in accordance with the Declaration of Helsinki.

\section{Experimental procedure}

At the beginning of the experiment, novices received a 20-min standardized introduction to cello bowing, approved by a highly experienced cello teacher (one of the authors, SP). This included instructions of how to hold the bow with the right hand, controlling the position and movement of the bow relative to the string, and controlling bowing velocity. In particular, novice participants were explicitly instructed to maintain an orthogonal angle and constant contact point between bow and string, and to move the bow at a constant velocity. For participants without any prior musical experience, additional instructions and practice were given regarding timing their own movements with the metronome. Special care was taken to prevent fatigue or injury in carrying out the unfamiliar movement, by suggesting trying to perform the movements with as little effort as possible, providing breaks, and asking participants to perform relaxing hand and arm movements between the trials.

Due to organizational constraints (laboratory availability and concurrent professional commitments), the experiment was carried out in two different laboratories (Berlin and Eugene, see author affiliations of JV and MW). One of the authors (MW) was present during the experiments in both laboratories, ensuring consistency of experimental procedures. Of the 20 participants, eight were tested in Berlin (6 novices, 2 experts) and twelve in Eugene (4 novices, 8 experts). Unfortunately, this means that Group (expert, novice) and Laboratory (Berlin, Eugene) are confounded variables. We dealt with this methodological issue conservatively, by assessing the effects of Group (experts, novices) only after accounting for effects of Laboratory (see Statistical Analysis). Kinematic data were acquired using 3D motion capture systems (Berlin: Vicon MX, Oxford, UK, sampling rate $120 \mathrm{~Hz}$; Eugene: Motion Analysis, PEAK Performance Technologies, Englewood, $\mathrm{CO}$, sampling rate $60 \mathrm{~Hz}$ ). The data acquired in Berlin were downsampled to $60 \mathrm{~Hz}$ during preprocessing.

Participants wore sleeveless shirts so that the shoulders were free for marker placement. Passive reflective markers (diameter $12 \mathrm{~mm}$ ) were attached directly on the skin of the participants on the trunk (sternum, C7), right arm (acromion, lateral epicondyle of the elbow, lower arm, and wrist), and right hand (first metacarpophalangeal joint and first proximal interphalangeal joint). Cello and bow motion were recorded with additional markers on the cello (scroll and tail piece, defining the "string axis," and on the cello body, defining the lateral axis) and on the bow (on the tip, and about two-thirds of the way between tip and frog), see Fig. 1.

The task consisted of repeated bowing movements on the open A-string (the string with the highest pitch, without using the left hand to change the pitch) at a metronomeprescribed tempo of 60 bows per minute, that is, 30 downbows and 30 up-bows. Participants were instructed to try to use the full length of the bow, beginning at the frog (where the bow is held with the right hand) and alternating downand up-bow movements. Twenty bowing cycles (up and down movements) were acquired per participant.

\section{Data analysis}

Kinematic data were resampled to the common sampling rate of $60 \mathrm{~Hz}$ and low-pass-filtered with a bidirectional fifth-order Butterworth filter with a cutoff frequency of $20 \mathrm{~Hz}$. The cutoff frequency was chosen to be well below the Nyquist frequency $(30 \mathrm{~Hz})$, in order to remove measurement noise while preserving potential high-frequency components at bow reversals. Based on anthropometric measures (elbow, wrist, and hand thickness) and information about the bow (bow length), approximate joint center and bow frog positions were computed and added as "virtual markers" for further analysis. Bow movement data were transformed to a cello-centered coordinated system to allow for analyzing movement of the bow relative to the string. The cello-centered coordinate system had axes aligned with the strings ( $\mathrm{X}$, from scroll to tailpiece marker), orthogonal to the strings and parallel to the front plane (Y), and perpendicular to the front plane $(\mathrm{Z})$.

The biomechanical model (Rab et al. 2002) comprised six body segments: trunk, shoulder complex, upper arm, lower arm, hand, and index finger (right side of the body); and 12 DOF (joint angles): sterno-clavicular joint (3 DOF), glenohumeral (shoulder) joint (3 DOF), elbow (2 DOF), wrist (2 DOF), and finger (first metacarpophalangeal) joint (2 DOF). Data inspection and information from the cello pedagogy literature indicated that the four joint angles mostly involved in producing the cyclical bow movements are: shoulder adduction/abduction, elbow flexion/extension, and wrist and finger flexion/extension (the lower arm was found to be pronated during bowing movements, such that the wrist flexion/extension axis tended to be parallel to the elbow flexion/extension axis). For these angles, positive values indicate abduction (shoulder) and flexion (elbow, wrist, and finger).

The position and orientation of the bow relative to the string were analyzed in three ways. First, the bowing angle was defined as the spatial angle between the cello's long axis $(\mathrm{X})$ and the bow's long axis (line through the two bow markers). Second, the touch of the bow on the string, along the long axis of the cello, was estimated by determining the 
Fig. 2 Segmentation of bow movements by a velocity (zero crossing) criterion. Bow position (upper panel) is the estimated bow-string contact point, expressed relative to the bow. Bow velocity (lower panel) is the three-point temporal derivative of bow position
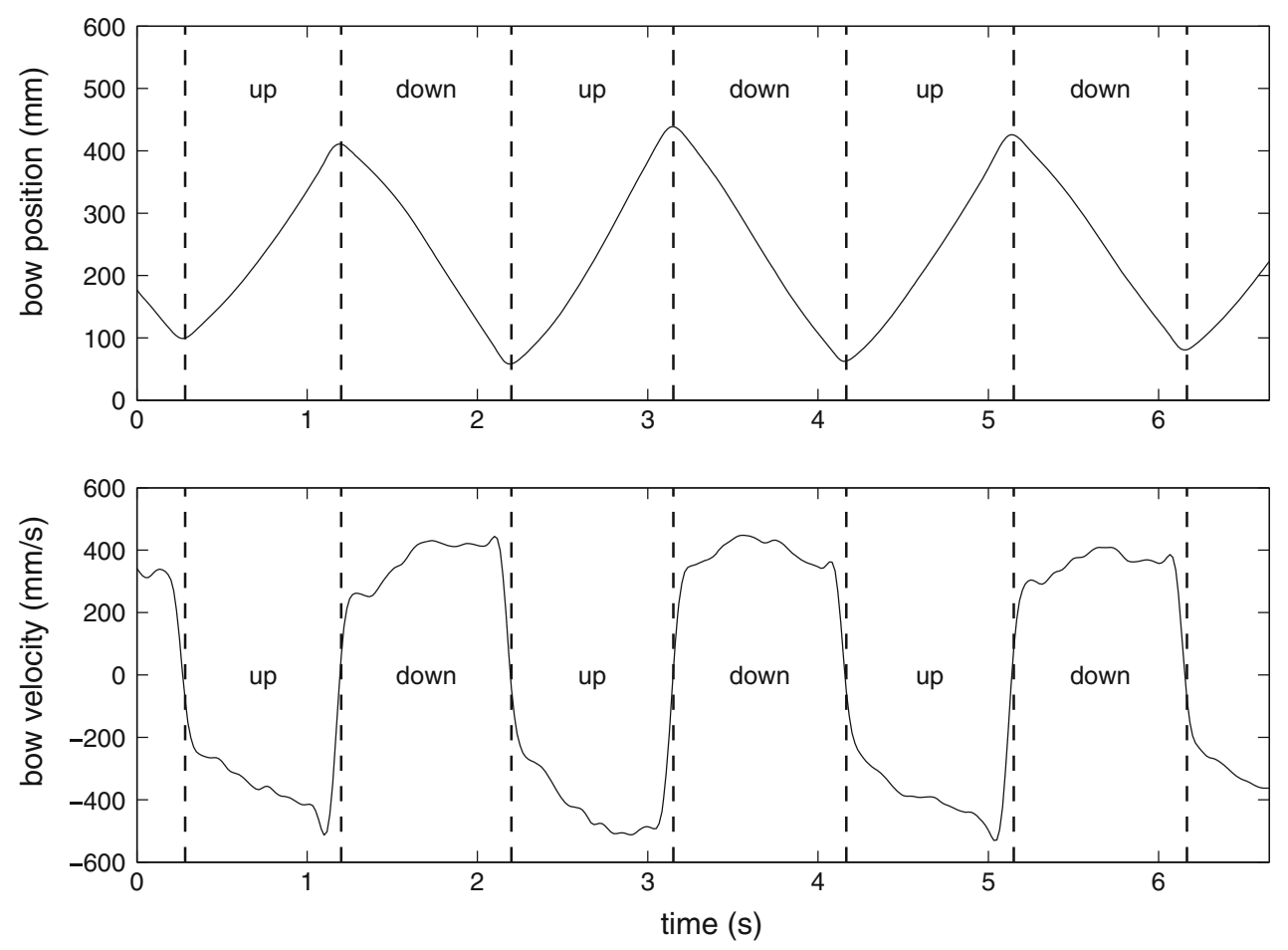

intersection of the line through the two bow markers with the cello's "sagittal" (XZ) plane. This was defined as the string touch position. Thus, larger values in string touch position correspond to a lower position on the cello. Third, the position of this bow touch point relative to the bow was defined as the bow touch position. The fact that the bowing was performed on the A-string, which is located about $2 \mathrm{~cm}$ outside the $\mathrm{XZ}$ plane, introduces inaccuracies in this analysis. Importantly, this does not affect the bowing angle, and due to the geometric layout (bow approximately orthogonal to the string), the error should be small for string touch position and constant (around $2 \mathrm{~cm}$ ) for bow touch position. For a deviating bowing angle of $\alpha=80^{\circ}$ (as sometimes found in the novices), the variable errors for string and bow touch position are estimated as $2 \mathrm{~cm} \times$ $\cos \alpha=0.35 \mathrm{~cm}$ and $2 \mathrm{~cm} \times(1-\sin \alpha)=0.03 \mathrm{~cm}$, respectively.

Bowing velocity was defined as the component of the velocity of the bow tip (3-point differentiation of the position of the second bow marker) parallel to the current bow orientation (line through the two bow markers), computed by the inner product of the two vectors. This definition is based on the fact that only movement along the bow's longitudinal axis leads to significant linear movement of the bow on the string, which produces the sound. Zero-crossings of bow velocity were used to identify bow movement reversals (changes in direction from up-bow to down-bow, see Fig. 2). Spatial and joint angle data were segmented according to bow reversals and time normalized to 100 data points for kinematic analysis within and between bows. Only movements between detected bow reversals with a duration between 0.5 and $1.5 \mathrm{~s}$ (instructed duration: $1 \mathrm{~s}$ ) were scored as bowing movements. Of these, only those without missing values (for bow position or joint angles) were considered $(92.6 \%$ of all bowing movements). To minimize the potential effects of different number of valid bowing movements between participants (and groups), only the first 10 up-bow and down-bow movements without missing values were used from each participant.

The use of degrees of freedom of the right arm was analyzed in two ways. First, the total amount of variability (expressed as SD) in each joint angle was determined as an indicator of how much movement occurred in this joint during bowing. Second, temporal coordination across joints was analyzed using principal component analysis (PCA, Daffertshofer et al. 2004). The amount of variance explained by the first principal component (PC1) was determined both in relation to the overall variability and for each joint separately. To this end, joint angle data (shoulder, elbow, wrist, and finger) from time-normalized bow movements (10 bow movements with 100 samples) were submitted to a principal component analysis (based on the covariance matrix of joint angles across time), resulting in component loadings and coefficient time series. From these, the proportion of individual and total joint variance explained by PC1 was computed by projecting the coefficient time series of PC1 back into joint angle space and comparing the variance (per joint angle or summing variance across joints) between original and reconstructed data. 
Both the proportion of explained variance (total variance and per joint angle) and the component loadings (per joint angle) were submitted to statistical analysis.

Dependent variables and statistical analysis

Based on the segmented and normalized bowing data, mean and SD of the bowing parameters (bow duration, bow amplitude, bowing angle, and string touch position) were determined as described above. Mean and SD of bowing velocity were determined based on the central (10-90\%) portion of the bowing movements, that is, away from the bow reversal. Variability measures were log-transformed prior to statistical analysis to correct for non-normal distribution.

The average amount of motion during a bow movement was determined for the four joint angles indicated above (shoulder, elbow, wrist, and finger). The proportion of variance explained across bowing movements by the first principal component was determined with respect to the total variance (all four joints) and for each joint separately. To correct for non-normal distribution, these measures were rescaled to the interval $[-1,1]$ and transformed by the inverse hyperbolic tangent. Component loadings of the first principal components were determined for each joint and submitted to statistical analysis.

Statistical comparisons were performed in $\mathrm{R}$ (Lawrence 2011; R Development Core Team 2008). To control for the confounding effect of Laboratory (Berlin, Eugene), statistical effects of Group and Bow Direction were analyzed after removing any main effects (mean differences) of Laboratory. After removing Laboratory effects, data were submitted to a two-way repeated measures ANOVA with Group (expert, novice) as between-subject factor and Bow Direction (up and down) as within-subject factor. Interaction effects were scrutinized by pairwise $t$ tests (Bonferroni-corrected). Statistical analyses were performed with a significance threshold of 0.05. Effect sizes for ANOVAs are reported as generalized eta-squared, $\eta^{2}$ (Bakeman 2005).

\section{Results}

Raw data

Figure $3 \mathrm{a}$ shows arm and bow postures at the times of successive bow reversal (10 up and 10 down reversals) in an expert (top) and novice (bottom), bowing at 60 BPM. Solid lines represent body segments and the bow, with the sternum and the tip of the bow marked by black circles. The axis of the string is indicated by the dotted vertical line. The 3D data were projected to the best approximating plane (determined by a PCA), which corresponded approximately to the XY-plane of the cello. Note the higher variability in the novice relative to the expert, both in bow positions and orientations, and in joint angles. Also note the increased use of the shoulder joint (larger shoulder angle differences between up- and down-bow end points) in the beginner, associated with substantial changes in bow angle in relation to the string, when comparing the up-bow to the down-bow end points.

Figure $3 b$ shows time-normalized sample data from the same two participants during a down-bow movement: bowing angle, string touch position, bow velocity, and joint angles (shoulder, elbow, wrist, and finger). The expert showed smaller variability, in particular for the bowing angle and the joint angles, and was closer to the "ideal" bowing angle of 90 degrees (Mantel 1995, p. 172) than the novice. The joint angle pattern of the expert also suggests larger movement in the elbow and wrist joint and (possibly) a higher degree of temporal coordination among the joint angles. This suggests that the novice is using the biomechanical DOF, in particular the distal joints, of the arm to a lesser extent than the expert during the bowing movement (see below).

\section{Experts show better control of bowing parameters}

Means and SDs of the bowing parameters are plotted in Figs. 4 and 5. The results of the statistical analysis (ANOVA) are shown in Table 1. Significant main effects of Group were found for the mean bowing angle and string touch position, indicating a lower bowing position on the string and a smaller deviation from a $90^{\circ}$-bowing angle in experts compared to novices. For the string touch position, main effects of Bow Direction and an interaction effect were also present. Post hoc analyses showed that experts (but not the novices) systematically varied string touch position between up- and down-bows $(p=0.022)$ and that the difference between experts and novices was significant both for up-bows ( $p=0.016)$ and down-bows ( $p=0.004)$.

For the variability measures (SD), significant main effects of Group were found for all bowing parameters, indicating in each case smaller variability in experts compared to novices.

Thus, experts showed better control of bowing parameters than novices, as indicated by a bowing angle closer to the instructed ("ideal") angle of $90^{\circ}$ and by reduced bowing variability for all parameters.

Experts show increased use and coordination of distal degrees of freedom

Joint angle amplitudes and coordination indices (component loadings and explained variance, PCA) are shown in 

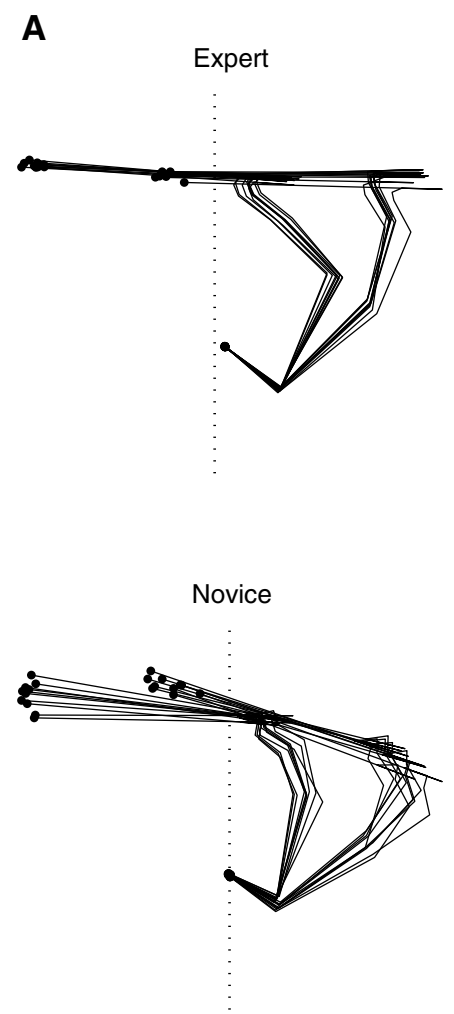

B
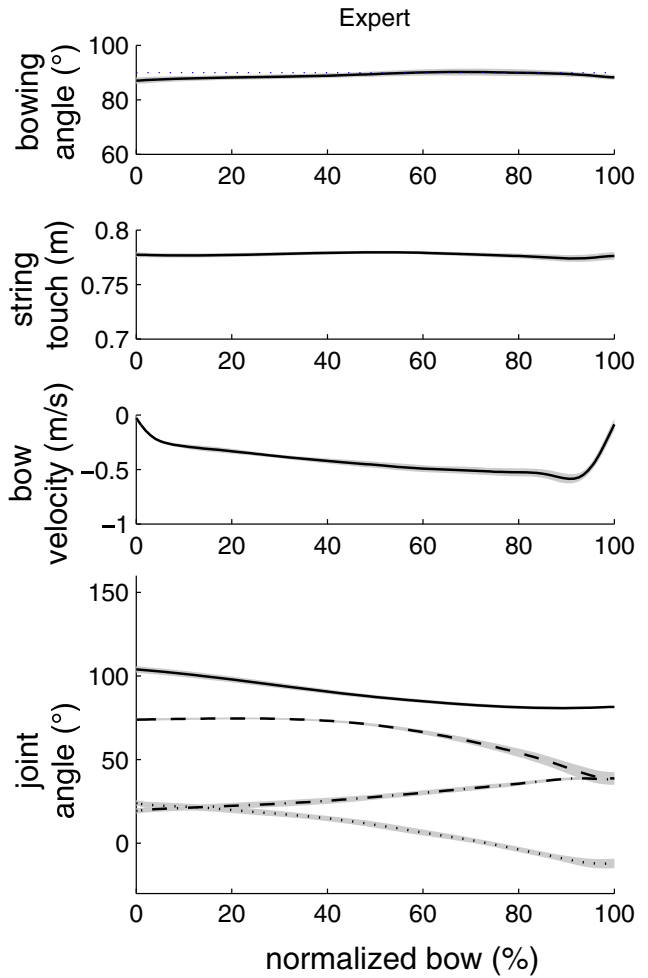
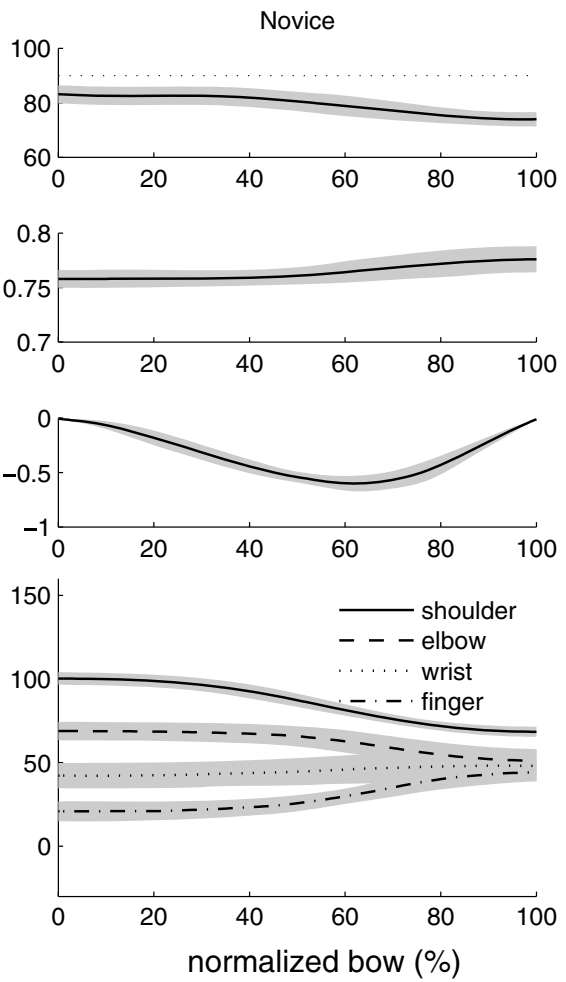

Fig. 3 a Arm postures at the times of bow reversal for an expert (upper panel) and novice (lower panel). b Time-normalized sample data (solid line: mean, gray shading: $\mathrm{SD}$ ) of expert (left) and novice (right): from top to bottom: bowing angle, string touch position, bow velocity, and joint angles
Fig. 4 Mean values for bow duration (a), bow angle deviation (b), string touch position (c), and bow velocity (d). Error bars represent SE. Statistical effects $(* p<0.05$, $* * p<0.01, * * * p<0.001)$ are indicated by symbols in the center (main effects of Group: *; Group $\times$ Direction interactions: $\mathrm{x}$ ) or above the bars (main effects of Bow Direction: *)
A

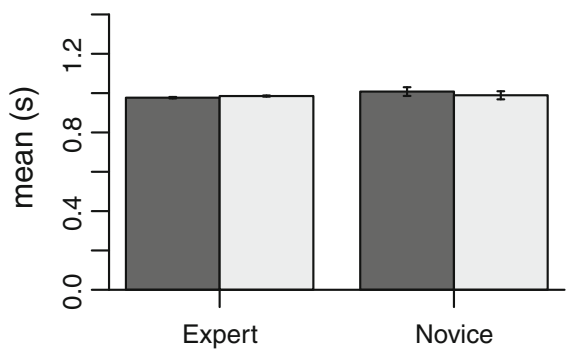

C

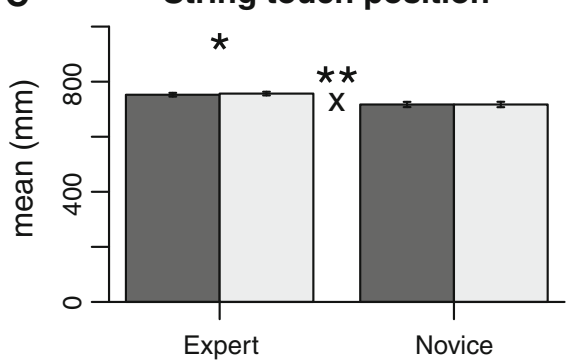

B Bow angle deviation

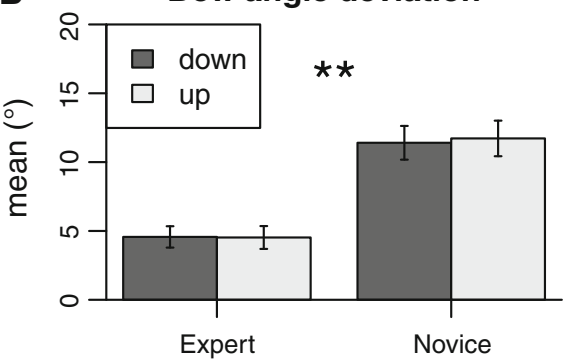

Bow velocity

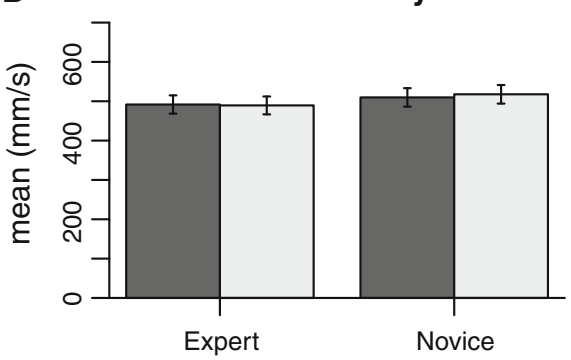

Figs. 6, 7, and 8. The results of the statistical analyses for these measures are listed in Table 2. For joint movement amplitudes, significant main effects of Group were found for the elbow and wrist joint, with greater joint movement in experts compared to novices. The interaction effect for wrist movement amplitude reflects a difference between up- and down-bows in experts $(p<0.001)$ but not in novices $(p=0.11)$. The difference between experts and 
Fig. 5 Variability scores for bow duration (a), bow angle deviation (b), string touch position (c), and bow velocity (d). Error bars represent SE. Statistical differences between the groups are indicated (see caption of Fig. 4)
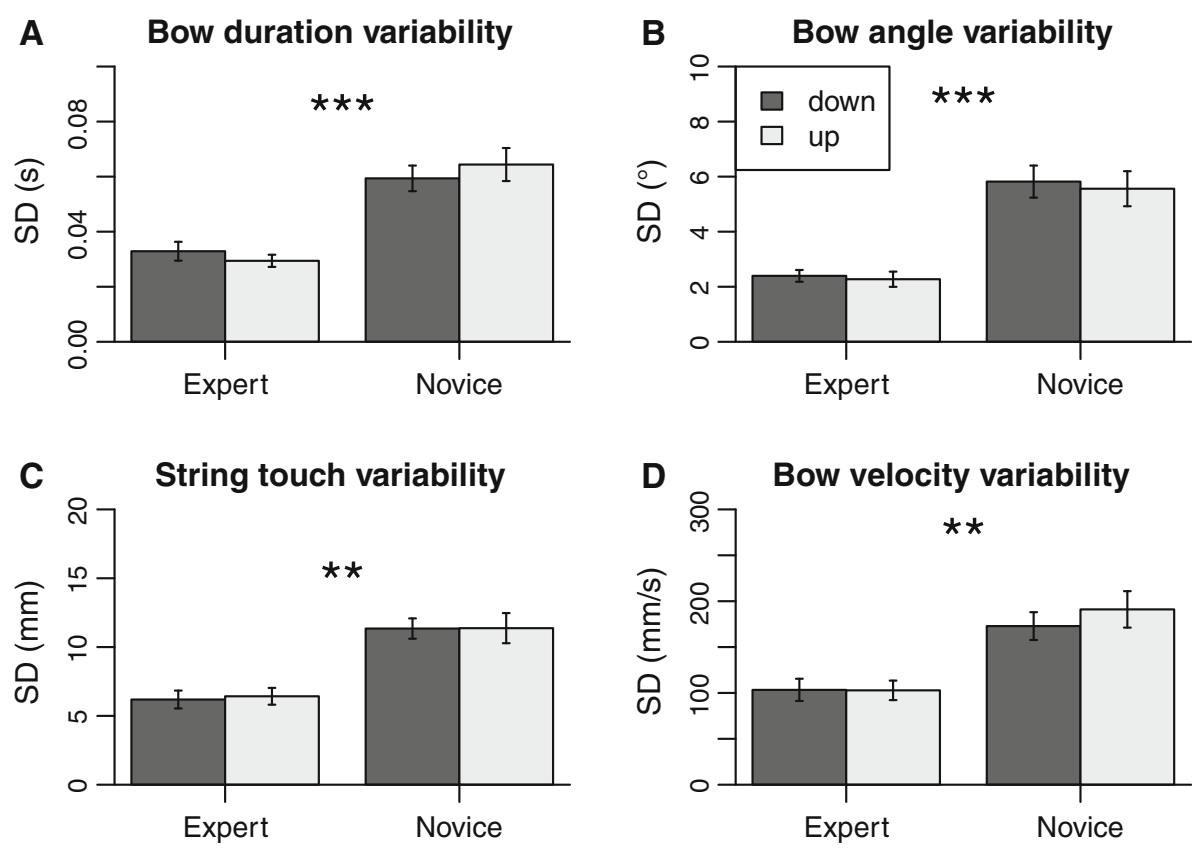

Table 1 Statistical results for bow parameters

\begin{tabular}{|c|c|c|c|c|}
\hline Variable & Measure & Group $^{b}$ & Bow Direction $^{\mathrm{b}}$ & Group $\times$ Direction $^{\mathrm{b}}$ \\
\hline Bow duration & $\begin{array}{l}\text { Mean } \\
\text { SD }\end{array}$ & $\begin{array}{l}F=0.16, p=0.693, \eta^{2}=0.01 \\
\boldsymbol{F}=\mathbf{2 3 . 8 8}, \boldsymbol{p}<\mathbf{0 . 0 0 1}, \boldsymbol{\eta}^{\mathbf{2}}=\mathbf{0 . 5 0}\end{array}$ & $\begin{array}{l}F=0.56, p=0.463, \eta^{2}=0.00 \\
F=0.02, p=0.887, \eta^{2}=0.00\end{array}$ & $\begin{array}{l}F=3.84, p=0.066, \eta^{2}=0.02 \\
F=1.07, p=0.315, \eta^{2}=0.01\end{array}$ \\
\hline Bowing angle & $\begin{array}{l}\text { Mean }^{\mathrm{a}} \\
\mathrm{SD}\end{array}$ & $\begin{array}{l}F=13.15, p=0.002, \eta^{2}=0.42 \\
F=20.70, p<0.001, \eta^{2}=0.51\end{array}$ & $\begin{array}{l}F=0.36, p=0.557, \eta^{2}=0.00 \\
F=1.72, p=0.206, \eta^{2}=0.01\end{array}$ & $\begin{array}{l}F=0.60, p=0.447, \eta^{2}=0.00 \\
F=0.00, p=0.949, \eta^{2}=0.00\end{array}$ \\
\hline String touch & $\begin{array}{l}\text { Mean } \\
\text { SD }\end{array}$ & $\begin{array}{l}F=11.93, p=0.003, \eta^{2}=0.39 \\
F=11.98, p=0.003, \eta^{2}=0.38\end{array}$ & $\begin{array}{l}\boldsymbol{F}=\mathbf{7 . 4 1}, \boldsymbol{p}=\mathbf{0 . 0 1 4}, \boldsymbol{\eta}^{2}=\mathbf{0 . 0 1} \\
F=0.09, p=0.764, \eta^{2}=0.00\end{array}$ & $\begin{array}{l}\boldsymbol{F}=\mathbf{7 . 5 2}, \boldsymbol{p}=\mathbf{0 . 0 1 3}, \boldsymbol{\eta}^{2}=\mathbf{0 . 0 1} \\
F=0.80, p=0.383, \eta^{2}=0.00\end{array}$ \\
\hline Bow velocity & $\begin{array}{l}\text { Mean } \\
\text { SD }\end{array}$ & $\begin{array}{l}F=0.18, p=0.679, \eta^{2}=0.01 \\
F=\mathbf{1 1 . 4 4}, \boldsymbol{p}=\mathbf{0 . 0 0 3}, \boldsymbol{\eta}^{\mathbf{2}}=\mathbf{0 . 3 7}\end{array}$ & $\begin{array}{l}F=0.53, p=0.475, \eta^{2}=0.00 \\
F=0.83, p=0.375, \eta^{2}=0.00\end{array}$ & $\begin{array}{l}F=1.78, p=0.198, \eta^{2}=0.00 \\
F=0.72, p=0.408, \eta^{2}=0.00\end{array}$ \\
\hline
\end{tabular}

a Mean deviation of bowing angle from $90^{\circ}$

${ }^{\text {b }} F=F(1,18), \eta^{2}=$ generalized eta-squared, significant effects $(p<0.05)$ in bold font

novices in wrist movement amplitude was significant for both bow directions (up: $p=0.002$ and down: $p=0.008$ ).

For the component loadings (Fig. 7), main effects of Group were found for shoulder (larger in novices), as well as elbow, wrist, and finger (larger in experts). For the shoulder joint, an additional interaction effect was found, but post hoc comparisons were not significant (all $p>0.1$ ). For the elbow and wrist joint, there were significant main effects of Bow Direction and interaction effects, with experts showing different loadings for up- and down-bows (elbow and wrist: $p<0.001$ ), while novices did not (elbow: $p=0.136$, wrist: $p=0.20$ ). The difference between experts and novices was significant only for downbows for the elbow (down: $p=0.026$, up: 0.07) and for both directions for the wrist (down: $p=0.008$, up: $p=0.002$ ).

With respect to the proportion of explained variance (Fig. 8), main effects of Group were found for the shoulder joint (larger in novices), and elbow and wrist joints (larger in experts). For the shoulder, the main effect of Bow Direction and the interaction effect were significant as well. Post hoc comparisons showed that the groups differed in this variable only for down-bows $(p=0.028)$, and that only experts showed a significant difference between up- and down-bows $(p=0.002)$. For wrist, an additional main effect of Bow Direction was found. Post hoc comparisons indicated, however, that this effect was only present in experts $(p=0.008)$ and confirmed that the difference between groups was significant for both bowing directions (up: $p<0.001$, down: $p=0.004$ ).

Finally, the total amount of variance explained by the first principal component (mean $\pm \mathrm{SD}$; experts/down: $88.5 \pm 6.6 \%$, experts/up: $92.9 \pm 3.6 \%$, novices/down: $85.8 \pm 8.5 \%$, novices/up: $85.5 \pm 8.8 \%$ ) exhibited a significant main effect of Bow Direction and a significant interaction. Post hoc comparisons showed that experts 
Fig. 6 Joint movement amplitudes for the four joint angles analyzed in this study: a shoulder adduction/abduction, b elbow flexion/extension, c wrist flexion/extension, and d finger flexion/extension. Error bars represent SE. Statistical differences between the groups are indicated (see caption of Fig. 4)
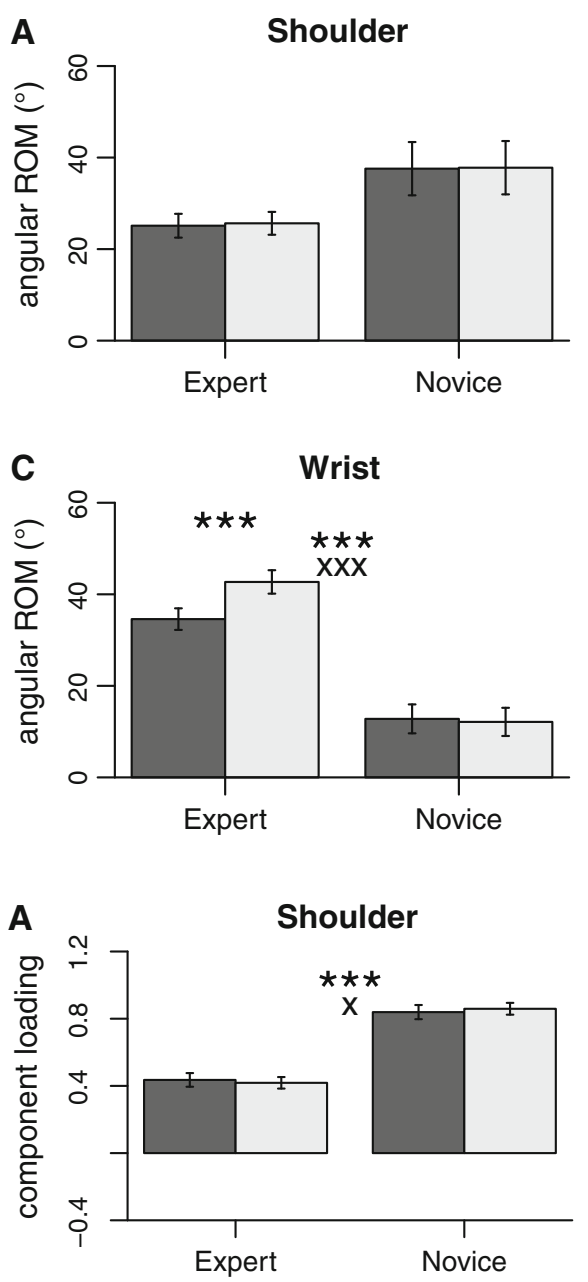

Fig. 7 Component loadings of the first principal component (PC1) on the four joint angles. Error bars represent SE.

Statistical differences between the groups are indicated (see caption of Fig. 4)
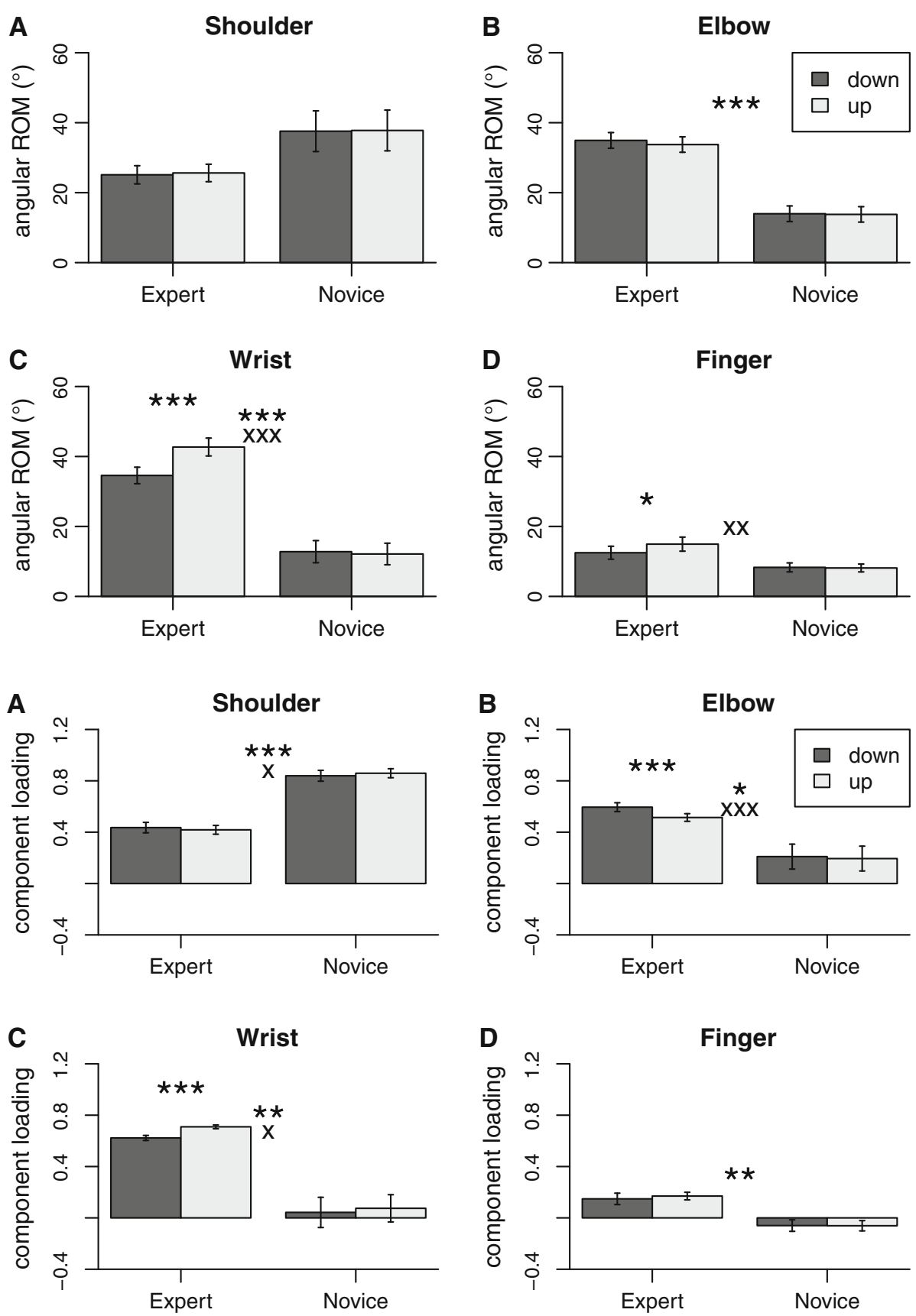

differed in this measure between up- and down-bows ( $p=0.002)$, and this was not the case in novices $(p>0.5)$.

Thus, in comparison with novices, experts made more (movement extent) and more coordinated (component loadings, variance explained by PC1) use of the elbow, wrist, and finger joints. In contrast, the shoulder exhibited smaller component loadings and a smaller proportion of explained variance in experts compared to novices. Taken together, these results suggest an expertise-related difference in the use of proximal (shoulder) versus more distal (elbow and wrist) degrees of freedom. In addition, some of the joint angle/coordination measures also exhibited interaction effects of Group and Bow Direction. Post hoc comparisons showed that these interaction effects reflected differences between up- and down-bow movements which were present in experts but not in novices.

\section{Discussion}

The aim of the present study was to test the applicability of Bernstein's theory of graded skill acquisition (Bernstein 1967) to cello bowing. We compared bowing movements and joint angle coordination in expert and novice cello 
Fig. 8 Joint angle variance accounted for by the first principal component (PC1) for the four joint angles. Error bars represent SE. Statistical differences between the groups are indicated (see caption of Fig. 4)
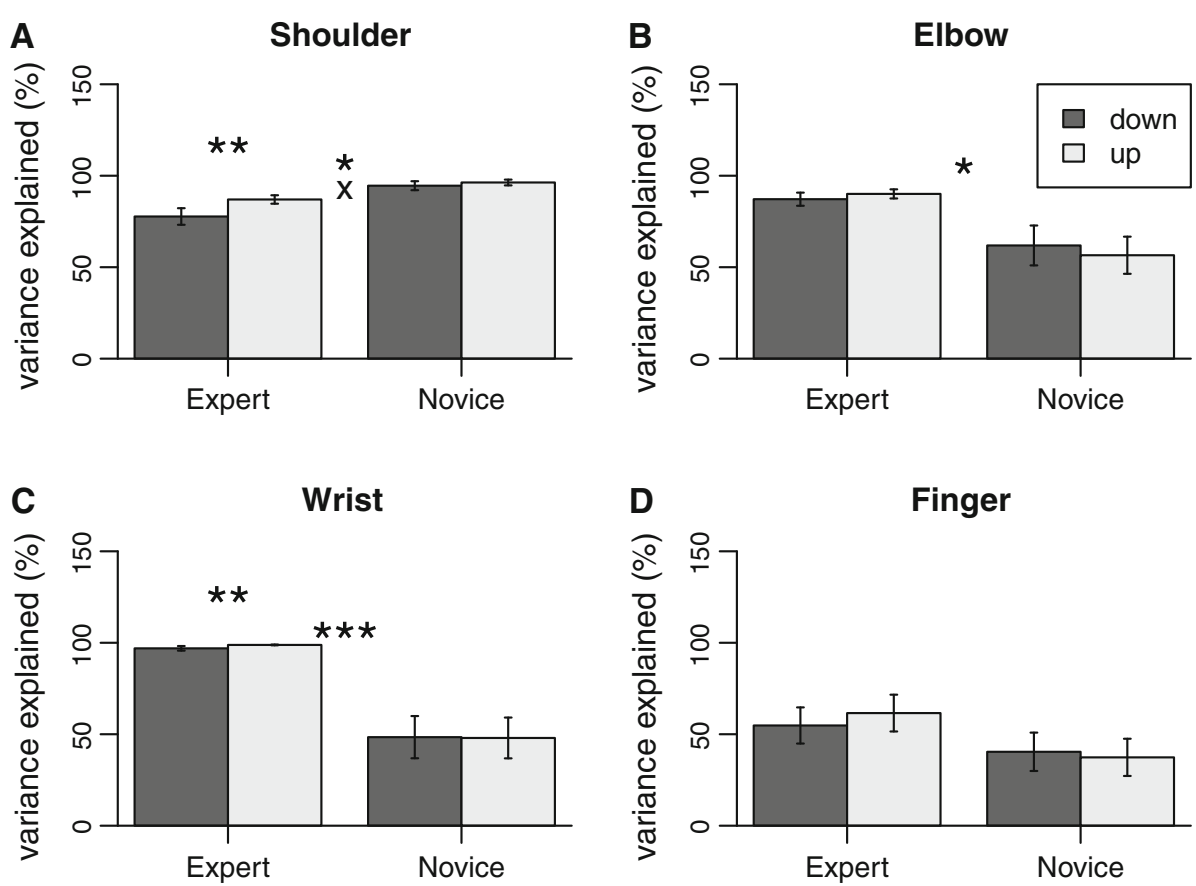

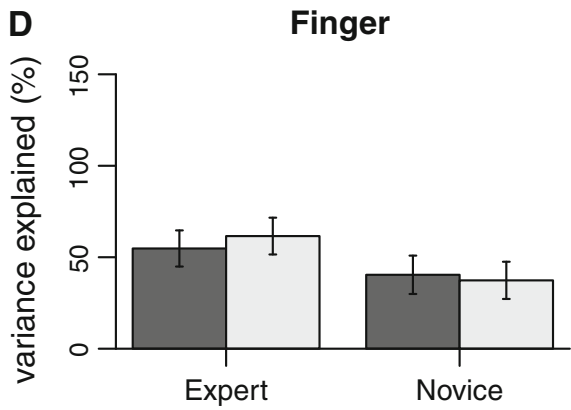

Table 2 Statistical results for joint angles

\begin{tabular}{|c|c|c|c|c|}
\hline Joint & Measure $^{a}$ & Group $^{b}$ & Direction $^{\mathrm{b}}$ & Group $\times$ Direction $^{\mathrm{b}}$ \\
\hline \multirow[t]{3}{*}{ Shoulder } & Amplitude & $F=2.18, p=0.157, \eta^{2}=0.11$ & $F=4.17, p=0.056, \eta^{2}=0.00$ & $F=0.75, p=0.397, \eta^{2}=0.00$ \\
\hline & Loading & $F=28.73, p<0.001, \eta^{2}=0.61$ & $F=0.02, p=0.878, \eta^{2}=0.00$ & $F=4.92, p=0.040, \eta^{2}=0.00$ \\
\hline & Expl.var. & $F=6.88, p=0.017, \eta^{2}=0.27$ & $F=24.66, p<0.001, \eta^{2}=0.02$ & $F=7.10, p=0.016, \eta^{2}=0.01$ \\
\hline \multirow[t]{3}{*}{ Elbow } & Amplitude & $F=24.19, p<0.001, \eta^{2}=0.57$ & $F=4.18, p=0.056, \eta^{2}=0.00$ & $F=2.23, p=0.153, \eta^{2}=0.00$ \\
\hline & Loading & $F=6.78, p=0.018, \eta^{2}=0.27$ & $F=35.12, p<0.001, \eta^{2}=0.01$ & $F=16.15, p=0.001, \eta^{2}=0.01$ \\
\hline & Expl.var. & $F=6.03, p=0.024, \eta^{2}=0.25$ & $F=0.09, p=0.770, \eta^{2}=0.00$ & $F=2.30, p=0.147, \eta^{2}=0.00$ \\
\hline \multirow[t]{3}{*}{ Wrist } & Amplitude & $F=21.67, p<0.001, \eta^{2}=0.54$ & $F=36.11, p<0.001, \eta^{2}=0.04$ & $F=50.10, p<0.001, \eta^{2}=0.05$ \\
\hline & Loading & $F=15.08, p=0.001, \eta^{2}=0.45$ & $F=33.02, p<0.001, \eta^{2}=0.01$ & $F=7.03, p=0.016, \eta^{2}=0.00$ \\
\hline & Expl.var. & $F=16.58, p=0.001, \eta^{2}=0.47$ & $F=7.79, p=0.012, \eta^{2}=0.02$ & $F=1.24, p=0.281, \eta^{2}=0.00$ \\
\hline \multirow[t]{3}{*}{ Finger } & Amplitude & $F=3.54, p=0.076, \eta^{2}=0.16$ & $F=9.22, p=0.007, \eta^{2}=0.01$ & $F=12.17, p=0.003, \eta^{2}=0.02$ \\
\hline & Loading & $F=8.92, p=0.008, \eta^{2}=0.32$ & $F=0.68, p=0.420, \eta^{2}=0.00$ & $F=0.85, p=0.369, \eta^{2}=0.00$ \\
\hline & Expl.var. & $F=0.00, p=0.996, \eta^{2}=0.00$ & $F=2.59, p=0.125, \eta^{2}=0.03$ & $F=0.53, p=0.477, \eta^{2}=0.01$ \\
\hline $\mathrm{All}^{\mathrm{c}}$ & Expl.var. & $F=1.52, p=0.234, \eta^{2}=0.08$ & $F=19.55, p<0.001, \eta^{2}=0.02$ & $F=22.76, p<0.001, \eta^{2}=0.03$ \\
\hline
\end{tabular}

${ }^{a}$ Joint angle amplitude, as well as component loadings and variance explained by the first principal component

${ }^{\mathrm{b}} F=F(1,18), \eta^{2}=$ generalized eta-squared, significant effects $(p<0.05)$ in bold font

c Total explained variance by the first principal component

players. As expected, novice cellists were less able to control important task variables, showing higher variability in bow duration, bowing angle, bow touch on the string, and bow amplitude, as well as a higher deviation of bowing angles from the "ideal" value of 90 degrees (Mantel 1995, p. 172). In addition, the analyses of joint angle movement (total movement amplitude, component loadings, and variance explained by the first principal component) showed that experts made more coordinated use of the more distal joints (elbow, wrist, and finger). Thus, better control of bowing parameters was associated with increased use and coordination of the right arm's DOF, indicating that "coordinated freeing," in particular of distal DOF, is a crucial component of the complex skill of cello bowing.

Control of bowing parameters

As expected, cello experts were better at controlling the bowing parameters analyzed in this study, both in terms of absolute value (bowing angle closer to $90^{\circ}$ ) and variability (timing, bowing angle, string touch, and bow velocity). Stabilization of these parameters was explicitly instructed 
to the novices in the present study. Hence, the fact that novices showed poorer control cannot be explained by lack of explicit knowledge of task requirements. Rather, it points at limitations of motor coordination and/or attentional capacities (simultaneously stabilizing and monitoring of multiple task variables). Clearly, the present study does not permit firm conclusions concerning the functional relevance of task variables such as bow velocity, string touch position, and bowing angle. Nevertheless, we would like to note the results are in agreement with the cello teaching literature, which claims that these variables should be controlled in skilled bowing (Mantel 1995). However, the fact that skilled cellists do control these variables (even without explicit instruction in the present experiment) may also be due to the fact that cello teachers emphasize these variables in their teaching and does not directly prove their functional relevance.

\section{Use and coordination of DOF}

As reviewed in the introduction, previous theoretical and empirical works (based on a variety of tasks) as well as the teaching literature (on the specific task of cello bowing) both suggest that novel complex motor skills are initially approximated by "freezing" degrees of freedom. This may be a way of reducing coordinative complexity at the expense of poorer and possibly less efficient performance (Bernstein 1967). As discussed in the Introduction, the term "freezing" still lacks a precise operationalization. In order to characterize the multi-DOF arm movement in detail, we quantified the use and coordination of the DOF both in terms of movement amplitude and temporal covariation among joint angles (analyzed using PCA, component loadings, and explained variance), in line with earlier studies on complex motor skills (Arutyunyan et al. 1969; Newell and Van Emmerik 1989; Vereijken et al. 1992), and extending an earlier study on violing bowing (Konczak et al. 2009).

Based on this analysis, we found evidence for reduced use and coordination of distal joints (wrist and elbow) in the novice compared to the expert participants. In contrast, a larger proportion of shoulder variance was explained by the first principal component in novices. These findings are corroborated by the component loadings of the first principal component, with larger loadings for the distal joints (elbow, wrist, and finger) in experts compared to novices. In other words, experts moved their distal joints in a way that was systematically organized (temporally coupled) in relation to the entire arm movement. In contrast, novices showed smaller or no evidence for systematic integration of these joints into the movement.

Interestingly, several of the joint angle measures (amplitude and coordination) indicate that experts coordinated the arms differently for up- and down-bows; for instance, the loading of the first principal component was larger for the elbow in down-bows and larger for the wrist in up-bows. No such effect was found in the novice participants. As, at the same time, experts showed lower variability in all bowing parameters, this strongly suggests that experts were exploiting motor-equivalence (achieving stable outcome with variable means, in this case joint configurations) to a larger extent than novices.

It is important to note that none of the novice participants in the present study had any manifest movement limitations. The fact that the use of the degrees of freedom differed so markedly between novices and experts is hence not explained by anatomical constraints but is likely due to actual differences in the ability to coordinate these DOF in a task-specific functional way. Thus, our results are in line with previous work on the skills of hand writing and racquetball performance (Newell and Van Emmerik 1989; Southard and Higgins 1987), as well as the literature on cello technique (Mantel 1995), indicating that higher stages of skill acquisition are characterized by more differentiated use of the DOF, freeing distal segments of the arm to allow more flexible and functional performance.

In a recent study, Konczak et al. (2009) reported evidence for increased "freezing" (reduced movement amplitudes) with advanced skill in violin bowing. Some important differences between the two studies need to be noted. First, we studied cello bowing and Konczak et al. studied violin bowing, the two instruments requiring different postures and hence biomechanical configurations. However, the string instrument literature suggests that the issues beginning players are facing when learning to bow are similar across different types of instruments, specifically regarding the flexible use of hand and wrist (Mantel 1995; Starr 1996). Methodologically, the two studies differ in that we report a more complete set of bowing parameters and joint angles: Konczak et al. (2009) reported only shoulder and elbow angles, as well as bowing angle. In particular, bowing amplitude was not controlled for in their study. The example videos put online by the research group (http://hsc.umn.edu/hsc-project3.html) suggest that the (novice) child was playing with substantially larger relative bow amplitude than the (expert) adult, which might explain some or all the observed differences in shoulder and elbow movement amplitudes. In addition, potential differences in wrist motion amplitude could not be determined due to technical difficulties in that study. In the present study (in which experts and novices did not differ in bowing amplitude), the wrist showed marked expertise effects, in line with the cello teaching literature. We therefore suspect that the conclusions of Konczak et al. (2009) are largely based on insufficient control of bowing parameters and measurement problems. The present results clearly indicate that bowing expertise (for the cello) is associated with increased use of the elbow and wrist joints. 


\section{Limitations}

Several limitations of the present study need to be addressed. First, due to organizational constraints, the effect Group (experts vs. novices) was confounded with Laboratory (Berlin, Eugene) in the present data. We dealt with this methodological problem by assessing Group differences in dependent variables only after accounting for any main effect of Laboratory. Despite this conservative approach, reliable differences between the expert and novice participants were found. Thus, if anything the present analysis might underestimate actual differences between the two groups.

Second, comparing experts to complete novices was to some extent a pragmatic decision, due to the challenge of finding an age-controlled sample of adult early beginners. Based on the present findings, we are unable to draw any conclusions regarding whether the differences we observed are due to the complete novelty of the task, and to what extent such differences would also be present in early beginners (with, say, several weeks of cello practice). Assessing cello bowing in novices also meant that only a very simple task could be used, limiting ecological validity of the study. Indeed, we do not claim to have completely described the complex skill of cello bowing, which involves much more refined technical and musical aspects (Baader et al. 2005; Winold and Thelen 1994). Thus, to gain a more complete characterization of this skill and its acquisition, longitudinal studies and studies involving more complex (and more musical) tasks are necessary.

Finally, from a methodological point of view, we used a relatively simple approach, involving mainly univariate measures of mean and variability at different levels (bow and joint kinematics). The observed expertise-related differences suggest that it was the increased use and coordination of joint angles observed in the experts which allowed them to achieve better control at the task level. However, it would be desirable to address the relationship between execution (joint angles) and performance (bow) more directly. Methods relating variability at these levels have been proposed (Müller and Sternad 2003; Scholz and Schöner 1999), based on a forward model mapping execution to performance variables. Unfortunately, the biomechanical complexity of the present task (for instance, the relationship between bow and hand cannot be modeled as an ordinary joint) renders definition of such a forward model difficult.

Outlook

Musical instrument performance depends on complex coordinative skills acquired through years of intense practice (Ericsson et al. 1993). In addition, music teachers often provide detailed accounts of "ideal" performance patterns, allowing the definition of performance criteria. Therefore, music performance represents an excellent model for studying acquisition of complex sensorimotor skills. Subsequent studies may exploit this basic characterization by assessing the skill longitudinally in early beginners, looking at specific interventions (for instance, targeting specific aspects of bowing), or the effect of attentional focus and dual-tasking (naturally present in real-world music performance). Of course, skilled cello performance is by no means limited to the simple cyclic tasks used in the present study-in fact, task complexity can easily be manipulated by including string changes (bowing on different strings), combining bowing and pitch control by the left hand (Baader et al. 2005; Chen et al. 2006, 2008), and varying rhythmic complexity or bowing style (Winold et al. 2002; Winold and Thelen 1994). Importantly, these experimental manipulations are not arbitrary additions to increase task difficulty but represent ecologically valid extensions or combinations of more elementary skills.

Acknowledgments We thank Magdalena Koschitzki, Nina Lisofsky, and Zahra Rezaie for their help with manual data processing, and Nina Lisofsky for helpful suggestions on an earlier version of the manuscript. We would also like to thank the reviewers for constructive comments and suggestions.

Conflict of interest The authors declare that they have no conflict of interest.

\section{References}

Arutyunyan G, Gurfinkel V, Mirskii M (1969) Organization of movements on execution by man of an exact postural task. Biophysics 14:1103-1107

Baader AP, Kazennikov O, Wiesendanger M (2005) Coordination of bowing and fingering in violin playing. Cogn Brain Res $23: 436-443$

Bakeman R (2005) Recommended effect size statistics for repeated measures designs. Behav Res Methods 37:379-384

Bernstein N (1967) The co-ordination and regulation of movements. Pergamon Press, Oxford

Bernstein N (1996) On dexterity and its development. In: Latash ML, Turvey MT (eds) Dexterity and its development. Lauwrence Erlbaum, Hillsdale, pp 3-244

Chen J, Woollacott M, Pologe S (2006) Accuracy and underlying mechanisms of shifting movements in cellists. Exp Brain Res 174:467-476

Chen J, Woollacott MH, Pologe S, Moore GP (2008) Pitch and space maps of skilled cellists: accuracy, variability, and error correction. Exp Brain Res 188:493-503

Daffertshofer A, Lamoth CJC, Meijer OG, Beek PJ (2004) PCA in studying coordination and variability: a tutorial. Clin Biomech 19:415-428

Ericsson KA, Krampe RT, Tesch-Römer C (1993) The role of deliberate practice in the acquisition of expert performance. Psychol Rev 100:363 
Konczak J, Velden HV, Jaeger L (2009) Learning to play the violin: motor control by freezing, not freeing degrees of freedom. J Mot Behav 41:243-252

Lawrence MA (2011) ez: easy analysis and visualization of factorial experiments. http://CRAN.R-project.org/package=ez. Accessed 2012

Mantel G (1995) Cello technique: principles and forms of movement. Indiana University Press, Bloomington

Müller H, Sternad D (2003) A randomization method for the calculation of covariation in multiple nonlinear relations: illustrated with the example of goal-directed movements. Biol Cybern 89:22-33

Newell KM, Van Emmerik REA (1989) The acquisition of coordination: preliminary analysis of learning to write. Hum Mov Sci 8:17-32

Rab G, Petuskey K, Bagley A (2002) A method for determination of upper extremity kinematics. Gait Posture 15:113-119
Scholz JP, Schöner G (1999) The uncontrolled manifold concept: identifying control variables for a functional task. Exp Brain Res 126:289-306

Southard D, Higgins T (1987) Changing movement patterns: effects of demonstration and practice. Res Q Exerc Sport 58:77-80

Starr W (1996) The Suzuki violinist: a guide for teachers and parents. Birch Tree Group Ltd

R Development Core Team (2008) R: a language and environment for statistical computing. Vienna, Austria. http://www.R-project.org/. Accessed 2012

Vereijken B, Van Emmerik RE, Whiting H, Newell KM (1992) Free(z) ing degrees of freedom in skill acquisition. J Motor Behav 24:133-142

Winold H, Thelen E (1994) Coordination and control in the bow arm movements of highly skilled cellists. Ecol Psychol 6:1-31

Winold H, Thelen E, Feng J (2002) On the rebound. The Strad 514-521 